\title{
Effects of proactive interference on rats' continuous nonmatching-to-sample performance
}

\author{
MICHAEL J. PONTECORVO \\ Indiana University, Bloomington, Indiana
}

\begin{abstract}
Rats performed a new delayed matching-to-sample task-the continuous nonmatching-tosample task. A variable number of trials with one stimulus alternated with trials with a second stimulus. A response on the trial following a stimulus change (nonmatch trial) was reinforced. Responses to repeated stimuli were never reinforced. Rats could maximize reinforcement by remembering across the intertrial interval which stimulus was presented on the previous trial. Sequential analysis indicated that interference from previous conflicting trials (proactive interference, PI) reduced response accuracy but did not affect retention: Accuracy was lower on trials following a nonmatch trial than on trials following repeated stimuli. Furthermore, accuracy increased as a function of the time between the to-be-remembered nonmatch trial and the previous interfering trial. However, neither time between trials nor the distance from a stimulus change affected the rate of decline in accuracy over the retention interval.
\end{abstract}

Contemporary theories of animal short-term memory are based predominantly on data from delayed matching-to-sample (DMS) tasks. Typically (e.g., D'Amato, 1973; Wasserman, 1976), a sample stimulus is briefly presented, then extinguished. After some delay, the subject must identify the sample from among one or more comparison stimuli. Retention is inferred from the percentage of correct choices as a function of the delay interval.

Relative to other delayed response tasks (e.g., Fletcher, 1965; Heise, 1975; Olton, 1978), the DMS paradigm offers two advantages for the study of memory in animals. First, it minimizes the importance of peripheral mediating responses. Since the subject is required to attend to discriminative stimuli both before and after the retention interval, the subject cannot "bridge" the retention interval simply by orienting toward the correct response alternative (e.g., Fletcher, 1965); the correct choice cannot be determined until after the comparison stimuli are presented. Second, in contrast to many delayed response tasks (e.g., delayed alternation), which require the subject to remember responses or response locations, DMS tasks may employ exteroceptive

This research was supported by Grant MH-14658-13 from the National Institute of Mental Health. Preparation of the manuscript was supported by Training Grant ES-07065 and Center Grant ES-00260 from the National Institute of Environmental Health Sciences. The author thanks Philip Bushnell, Steven Daniel, Hugh Evans, George Heise, and David Spencer for advice and comments, and Susan Hull and Eleanor Cordisco for help in preparation of this manuscript. A partial description of this work was presented at the meeting of the Midwestern Psychological Association, Detroit, Michigan, May 1981. Reprints can be obtained from the author at: Room 119, Building 56B, Lederle Laboratories, Pearl River, New York 10965. stimuli that permit a rigorous manipulation of stimulus parameters and precise specification of stimulus and retention interval duration. Thus, DMS has become the preferred paradigm for the study of animal short-term memory.

Because of their proficiency in performing DMS tasks, pigeons and monkeys have been the most popular subjects for studies of animal short-term memory. However, in recent years there has been an increasing interest in using DMS tasks to study memory in other animal species (e.g., Herman \& Gordon, 1974; Wallace, Steinert, Scobie, \& Spear, 1980). This research has been motivated both by an interest in between-species comparisons and by a desire to capitalize on the unique advantages (e.g., genetic purity, evolutionary importance, suitability for physiological research) that particular species offer for the study of the biological basis of behavior. This paper introduces a new form of the delayed matching-tosample paradigm, the continuous nonmatching-tosample (CNM) task, that can be used to study shortterm memory in rats. The CNM is a variant of the go/no-go matching-to-sample task introduced by Konorski (1959; Chorazyna, 1959) and recently used with rats by Wallace et al. (1980; see also Bradley \& Roberts, 1965; Stepien \& Cordeau, 1960; Wasserman, 1976). In the CNM procedure, a variable number of trials with one stimulus alternates with a variable number of trials with a second stimulus. The first leverpress on a trial following a stimulus change is reinforced. Leverpresses to repeated stimuli are never reinforced. Retention is inferred from the percentage of correct responses as the intertrial interval (retention interval) increases.

The CNM task differs from traditional DMS tasks in that trial stimuli in the CNM task are continuously 
related, rather than paired. In the $\mathrm{CNM}$, each trial stimulus serves both as a comparison stimulus (with respect to the preceding trial) and as a sample stimulus (with respect to the following trial). This continuous pairing of trials helps emphasize the essential features of the nonmatching-to-sample problem: repeated nonreinforced presentations of a single stimulus (match trials) are juxtaposed with a reinforced presentation of a new stimulus (a nonmatch trial). Thus, stimulus change (as opposed to particular stimuli or response patterns) is highlighted as the unique cue for reinforcement. Acquisition and performance of the CNM task might therefore be expected to exceed the performance in an analogous paired-trial matching-to-sample task.

The experiments presented here were designed both to provide parametric information on CNM performance and to examine the nature of the psychological processes involved in short-term memory in rats. Of particular interest were the effects of interference from previous trials (proactive interference, PI) on the time course of retention.

Studies of PI have repeatedly demonstrated that previous conflicting stimuli can reduce the accuracy of responding in short-term-memory tasks (Grant \& Roberts, 1973; Moise, 1976; Nelson \& Wasserman, 1978). There is no consensus, however, concerning the relationship between PI and the time course of retention. Temporal discrimination theories (D'Amato, 1973; Mason \& Wilson, 1974) hold that PI should be a primary determinant of response accuracy and of the time course of retention. Such theories make little provision for information loss or decay. Forgetting is attributed to temporal confusion, that is, an inability to determine which of the possible stimuli was presented most recently. Thus, temporal discrimination theories predict that the rate of forgetting should depend on the amount of confusion caused by previous conflicting stimuli. Although not inconsistent with the notion that PI can affect response accuracy and retention, alternative theories (Grant, 1981a; Honig, 1978; Roberts \& Grant, 1976) emphasize the importance of other factors (trace decay, individual encoding, or rehearsal strategies) in determining the time course of retention.

Tests of these theories have yielded conflicting results. Although some studies have found that PI can influence the rate of decline in accuracy over time (Grant, 1975; Jarvik, Goldfarb, \& Carley, 1969; Zentall \& Hogan, 1974), others have reported that PI disrupted response accuracy equally at all retention intervals (Grant, 1980; Grant \& Roberts, 1973; Moise, 1976; Worsham, 1975). Studies with rats have generally fallen into the latter category, demonstrating that PI influences response accuracy, but not the time course of retention (Christoph, 1976; Gordon, Brennan, \& Schlesinger, 1976; Grant, 1980). However, these latter studies have been limited to delayed- response (delayed-alternation) procedures. As noted above, delayed-response performance may be confounded by the effects of mediational responding during the retention interval and, thus, may not provide a precise measure of memory processes. Furthermore, a number of results suggest that delayed matching-to-sample tasks, such as the present CNM task, might be more sensitive to the effects of PI on retention (Fletcher \& Davis, 1965; Honig, 1978; Medin \& Davis, 1974; Spear, 1978).

The CNM task is particularly well suited for the study of interference from previous conflicting stimuli. In the CNM task, the effects of previous stimuli can be examined simply by comparing performance on trials following a stimulus change to performance on trials following a series of identical stimuli. Thus, PI can be studied without altering the normal experimental protocol. It is not necessary to present additional stimuli prior to the to-be-remembered stimulus (cf. Grant \& Roberts, 1973; Zentall \& Hogan, 1974). Furthermore, since only a single stimulus is presented on each trial of the CNM task, the source of interference can be more precisely specified. It is not necessary to separate the effects of the previous trial's sample and comparison stimuli, as would be the case with paired-trial DMS procedures (e.g., Grant, 1975; Moise, 1976). Finally, it is possible to compare the effects obtained in the CNM task with the effects obtained in a parametrically similar, nonmemory, discrimination task. Thus, it is possible to evaluate potential confounding effects of temporal and reinforcement-related factors (e.g., D'Amato \& O'Neill, 1970; Roberts, 1980) and to separate these effects from the effects of PI.

Experiment 1 examined the acquisition of the CNM task by rats. Experiment 2 examined the effects of PI on response accuracy and on the time course of retention. Experiment 3 utilized an analogous discrimination task to control for possible nonspecific effects in Experiment 2.

\section{EXPERIMENT 1}

\section{Method}

Subjects. Six naive 90-day-old male Sprague-Dawley rats, obtained from Laboratory Supply Co., Indianapolis, were housed individually with free access to food in their home cages. The rats were water deprived except for the $15-20 \mathrm{~min}$ immediately following each experimental session.

Apparatus. Stainless steel operant chambers $(25 \times 18.5 \times$ $24 \mathrm{~cm}$ ) were enclosed in soundproof shells. Two glass levers were mounted on the front panel of each chamber, $8.0 \mathrm{~cm}$ above the floor and $13.5 \mathrm{~cm}$ apart (center to center). The left lever was covered by a stainless steel cap and was not accessible to the rats. The right lever could be operated by a force of $25-35 \mathrm{~g}$. A water dispenser, centered on the front panel $3.25 \mathrm{~cm}$ above the floor, delivered an $0.05-\mathrm{ml}$ drop of water. The discriminative stimuli for Experiment 1 were produced by illuminating a translucent lens, centered $14.0 \mathrm{~cm}$ above the floor, with either a bright or a dim light. The intensity ratio of the stimuli (dim/bright) was approximately 0.04 . No other illumination was present during the session. All events 
were controlled and the data recorded by minicomputer (Texas Instruments, Model 980A).

Procedure. The rats were first shaped to leverpress, then allowed to leverpress for continuous reinforcement during four 1-h sessions. The rats were then trained to perform the CNM task illustrated in Figure 1. A variable number of trials with the bright light alternated with a variable number of trials with the dim light. The first leverpress on a trial following a stimulus change (the first leverpress on a "nonmatch" trial) terminated the trial and produced water reinforcement. Leverpresses to repeated stimuli (leverpresses on "match" trials) did not terminate the trial, were not reinforced, and caused the match trial to be repeated (a "correction" trial). The contingencies on a correction trial were identical to those on an initial match trial. However, performance on correction trials was recorded separately from that on initial trials. Failure to respond on either match or nonmatch trials had no scheduled consequence.

In Experiment 1 there were 100 nonmatch trials and, not counting correction trials, 358 match trials per session: on the average there were 3.6 match trials between successive nonmatch trials (range 0-11). Each stimulus (bright or dim light) appeared on approximately one-half of the match and one-half of the nonmatch trials. The maximum stimulus (trial) duration was $5 \mathrm{sec}$. The retention interval (intertrial interval) was fixed at $1 \mathrm{sec}$ during the first stage (Days 1-23), $2.5 \mathrm{sec}$ during the second stage (Days 2431 ), and 5 sec during the last stage (Days 32-90) of Experiment 1. A pretrial delay contingency was in effect during the last $1 \mathrm{sec}$ of the retention interval; a response during the pretrial delay postponed the onset of the next trial for an additional $1 \mathrm{sec}$. (This latter contingency was included to prevent response bursts that were initiated during the retention interval from being counted as trial responses, and also to minimize inappropriate retention-interval responding).

\section{Results}

The probability of a correct response on a nonmatch trial $[\mathrm{P}(\mathrm{Hit})]$ and the probability of an incorrect response on a match trial [false alarm: P(FA)] were calculated daily for each rat. The bottom panel of Figure 2 shows the daily values of $P(H i t)$ and $P(F A)$ averaged across rats. The pattern of acquisition shown in Figure 2 is representative of the pattern found for individual animals throughout the experiments in this paper. The rats typically exhibited a period of chance performance $[P(H i t)=P(F A)]$, followed by a period of rapid acquisition, during which $\mathrm{P}(\mathrm{Hit})$ increased and/or $\mathrm{P}(\mathrm{FA})$ decreased. The top panels of Figure 2 show $P($ Hit) and $P(F A)$ for the

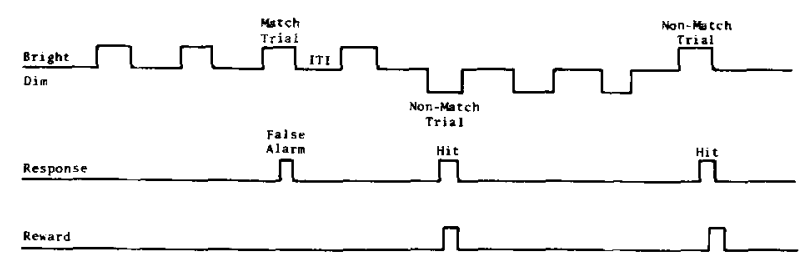

Figure 1. The continuous nonmatching-to-sample (CNM) procedure, showing a typical sequence of trials (top line), a possible pattern of responses (middle line), and reinforcement contingencies (bottom line). Responses on nonmatch trials ("Hits") were reinforced; responses on match trials ("False Alarms") were never reinforced. The intertrial interval (ITI) served as a retention interval.

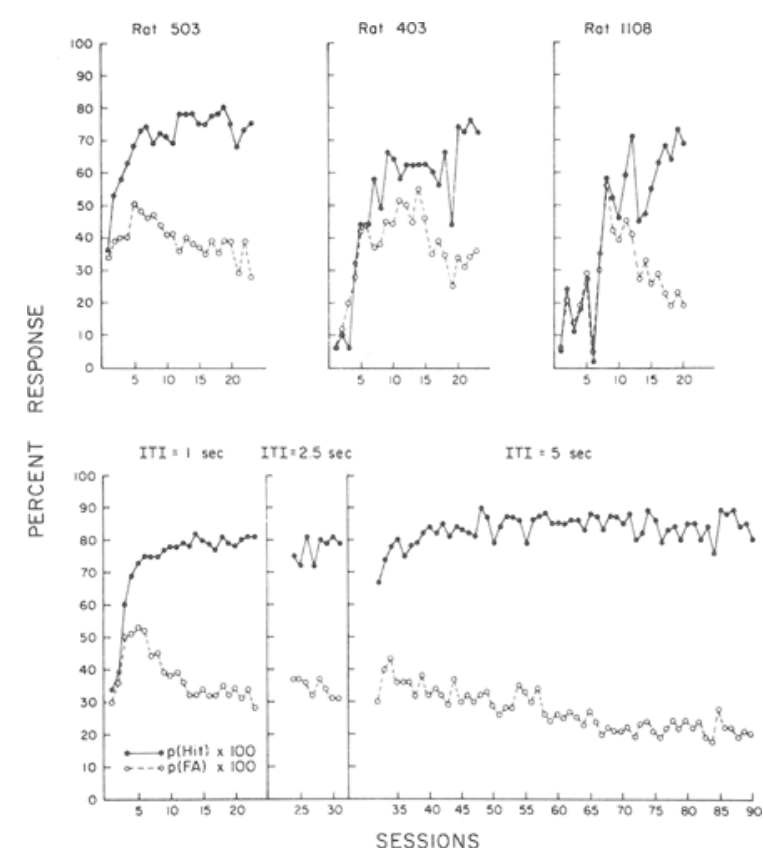

Figure 2. Acquisition and performance of the CNM task. Bottom: mean (group) performance in Experiment 1 with retention intervals (ITIs) of 1, 2.5, and 5 sec. Top: Performance of representative animals during the first stage (retention interval = 1 sec) of acquisition in Experiments 1 and 2.

first phase of acquisition (retention interval $=1 \mathrm{sec}$ ) for individual animals from Experiments 1 and 2 of this paper.

The total number of responses during the retention interval and the probability of an incorrect response on a correction trial were also calculated daily. These data were averaged for each animal over the last 25 days of the experiment (Days 66-90), since performance was most stable over this period. The average number of retention-interval responses ranged from 4 (Rat 501) to 51 (Rat 504) with a mean of 22. Assuming a maximum distribution of responses over intervals, these results indicate that responses occurred in fewer than $5 \%$ of the retention intervals. The probability of a correction-trial error during the last 25 days of Experiment 1 averaged 0.12 . Thus, responding was predominantly limited to initial trials, and in particular to the reinforced nonmatch trials.

\section{Discussion}

Experiment 1 showed that rats can readily solve the CNM task and perform consistently over the course of 90 sessions. Thus, Experiment 1 established the feasibility of this task as a baseline for studying the psychological or physiological determinants of short-term memory. However, there is one potentially important drawback to the CNM procedure used in Experiment 1. The task employs only one response manipulandum, with the subject's choices 
indicated by the occurrence or nonoccurrence of a response. Thus, it may be difficult to differentiate nonresponses resulting from disinterest or loss of motivation from nonresponses that indicate a discriminated "no-go" choice. The obvious solution to this problem is to modify the task to include a second response lever, such that responses on one lever are reinforced on nonmatch trials and responses on the second lever are reinforced on match trials. Unfortunately, however, pilot studies in our lab suggest that the use of a two-alternative (go-go) procedure may increase response variability and greatly lengthen the course of acquisition.

A less elegant but more practical solution is to utilize the theory of signal detection (TSD: Green \& Swets, 1966) to obtain separate estimates of response accuracy (sensitivity) and of the overall tendency to respond (bias). Although TSD analysis cannot eliminate the potential confounding by motivational or other nonspecific factors, it does help to alert the experimenter to the presence of such effects. Thus, if a treatment that decreases sensitivity also produces a large decrease in bias (response probability falls), then the experimenter should entertain the hypothesis that the treatment decreased motivation instead of, or in addition to, discrimination accuracy. On the other hand, if a treatment alters sensitivity but has little or no effect on bias, then the experimenter is relatively safe in attributing the treatment effects to changes in discriminative processes. For these reasons, TSD analysis was utilized in both of the experiments presented below.

\section{EXPERIMENT 2}

Experiment 2 examined the effects of proactive interference (PI) on the time course of retention in the CNM task. Rats were trained to perform a version of the CNM task in which the duration of the retention interval varied within each session among the values 2.5, 5, and $10 \mathrm{sec}$ (see Figure 3). Performance was measured as a function of the duration of the retention interval and the number of trials from the last nonmatch trial. Since the stimulus presented on the trial prior to a nonmatch trial (Trial NM-1) must, by definition, differ from the stimulus presented on the nonmatch trial, memory for this trial can be expected to interfere with the memory for the nonmatch trial. This interference should be evidenced by poor performance on the trial immediately following a nonmatch trial (Trial $\mathrm{NM}+1$ ), where the nonmatch trial stimulus must be recalled. In contrast, performance on the remaining trials of the session (Trials $\mathrm{NM}+2, \mathrm{NM}+3$, etc.) can be expected to be relatively free of PI because, for these trials, one or more repetitions of the same stimulus always separate the to-be-remembered stimulus from the most recent conflicting stimulus. Thus, response

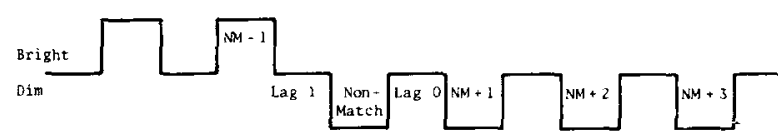

Figure 3. Possible trial sequence showing proactive interference as measured in Experiment 2. NM: $A$ nonmatch trial. NM-1: Trial prior to the nonmatch trial: the previous interfering stimulus. Lag 1: The interval between trial $N M-1$ and the nonmatch trial. NM+1: The trial following the nonmatch trial. Lag 0: The retention interval, in this case the interval between the nonmatch trial and Trial $\mathbf{N M + 1}$. Trial $N M-1$ was expected to interfere with performance on later trials. The magnitude of this effect was expected to depend on Lag 1 and the distance of the test trial $(N M+1$, NM+2) from Trial NM-1.

accuracy should be low on trials immediately following a nonmatch trial and increase for a number of trials thereafter. Similarly, if PI affects retention, then the rate of forgetting on NM+1 trials should be more rapid than the rate of forgetting on the remaining trials of the session. To the extent that PI is the primary determinant of the time course of retention, the rate of forgetting on these latter, low PI trials should be minimal. Alternatively, if other factors (e.g., decay, processing strategies) are also important, significant forgetting would be expected even at the largest distances from the previous conflicting stimulus.

Performance was also measured as a function of the interval (Lag 1) between a nonmatch trial and the previous conflicting trial (Trial NM-1). If, as suggested above, performance on $\mathrm{NM}+1$ trials is disrupted by interference from previous conflicting trials, then accuracy on $\mathrm{NM}+1$ trials should increase as a function of the time between the conflicting NM-1 trials and the to-be-remembered nonmatch trial. To the extent that PI also influences retention, the rate of retention should also increase (forgetting should decrease) as a function of the time between Trial NM-1 and the nonmatch trial.

\section{Method}

Subjects. Five naive, 90-day-old, male Sprague-Dawley rats, obtained from Harlan Industries, Inc., Indianapolis, were used as subjects in Experiment 2.

Apparatus. The apparatus was the same as that used in Experiment 1.

Procedure. All rats were shaped to leverpress, then trained to perform a CNM task similar to that in Experiment 1. As in Experiment 1 , there were 100 nonmatch and 358 match trials per session. The retention interval was fixed at $1 \mathrm{sec}$ during the first stage, $2.5 \mathrm{sec}$ during the second stage, and $5 \mathrm{sec}$ during the third stage of acquisition. In contrast to Experiment 1, subjects in Experiment 2 were required to achieve a minimum performance criterion before proceeding to the next stage of training. This criterion allowed subjects to complete the acquisition phase rapidly, while guaranteeing a minimum level of performance for all subjects. The retention interval was extended from 1 to $2.5 \mathrm{sec}$ when a subject had completed a minimum of 20 days' training with a mean sensitivity ( $\mathrm{A}^{\prime}$, Pollack \& Norman, 1964) of at least 0.80 for the last 5 days, or a minimum of 30 days' training with a 5-day mean $A^{\prime}$ of at least 0.75 . In the 2.5 - and 5 -sec stages, the rats were 
required to achieve a mean $A^{\prime}$ of at least 0.80 for 5 days or to complete 10 days in that condition before progressing to the next condition. Thus, acquisition required 30 to 50 days.

Following acquisition, all subjects were trained to perform a CNM task in which the retention intervals varied within each session among the values $2.5,5$, and $10 \mathrm{sec}$. The order of stimuli and retention intervals was counterbalanced across sessions, with each retention interval occurring on about $33 \%$ of the trials. This multiple-retention-interval condition lasted 36 sessions. All data analyses were based on the last 16 of these sessions.

\section{Results}

$\mathrm{P}(\mathrm{Hit})$ and $\mathrm{P}(\mathrm{FA})$ were calculated daily for each rat as in Experiment 1. These values were then used to compute indices of sensitivity and bias. Since the assumptions required by parametric models could not be verified, nonparametric indices of sensitivity (A': Pollack \& Norman, 1964) and bias (B'": Hodos, 1970) were calculated according to the formulas given by Grier (1971). A' can vary in value between 0.0 and 1.0 , with a value of 0.50 indicating chance responding and 1.0 indicating perfect accuracy. $\mathrm{B}^{\prime \prime}$ varies between -1.0 and 1.0 , with negative values indicating a bias to respond (i.e., a "yes" bias) and positive values indicating a bias against responding (i.e., a "no" bias). All statistical analyses were based on the values $\mathrm{A}^{\prime}$ and $\mathrm{B}^{\prime}$, with a probability value of $\mathrm{p}<.05$ required to demonstrate significance.

Multiple-retention-interval performance. $P(F A)$ increased, but $P(H i t)$ remained roughly constant as the retention interval increased from 2.5 to $10 \mathrm{sec}$ (Figure 4). Analysis of the corresponding values of $\mathbf{A}^{\prime}$ and $\mathbf{B}^{\prime \prime}$ indicated that $\mathbf{A}^{\prime}$ decreased significantly as the retention interval increased [mean $\mathrm{A}^{\prime}=0.79$, 0.81 , and 0.72 , respectively; $F(2,8)=14.40$ ]. $B^{\prime \prime}$ also decreased (the tendency to respond increased) with retention interval duration $\left[\mathrm{B}^{\prime \prime}=0.10,-0.14\right.$ and -0.12 , respectively; $F(2,8)=13.61]$.

Effects of previous conflicting stimuli. The number of response opportunities and the number of responses on match and nonmatch trials were summed for each rat as a function of trial type, the duration of the retention interval (Lag 0 ) and the number of trials, incliding correction trials, from the last nonmatch trial. Since there was no programmed limit on the number of correction trials, it was necessary to establish a cutoff value for this latter index. Accordingly, for each retention interval, trials more than 14 trials from the last nonmatch trial were summed into a single category, nonmatch +15 $(\mathrm{NM}+15)$.

Performance on trials following a nonmatch trial (NM +1 trials) was further analyzed by separately recording response opportunities and responses as a function of the duration of the interval (Lag 1) between the nonmatch trial and the previous conflicting stimulus. This analysis centered only on the longest $(10 \mathrm{sec})$ and shortest $(2.5 \mathrm{sec})$ values of Lag 1 and Lag 0 , because these values seemed most likely to

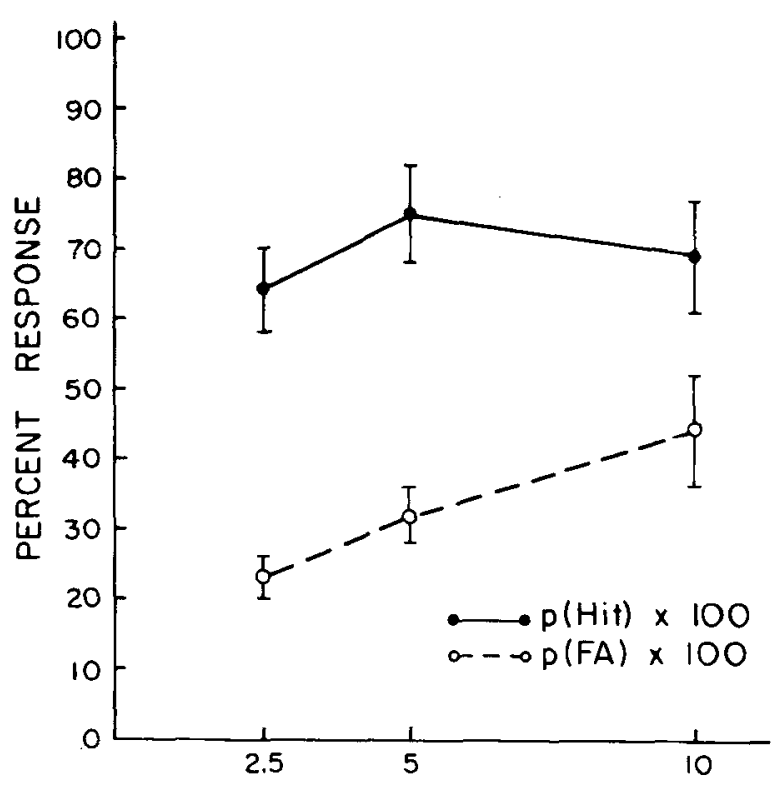

RETENTION INTERVAL (sec)

Figure 4. Mean (group) values of $P(H i t)$ and $P(F A)$ as a function of retention interval duration for the last 16 days of Experiment 2. Bars show standard error for each mean.

reveal differences in performance as a function of Lag 1.

Values of $\mathbf{P}(\mathrm{Hit}), \mathrm{P}(\mathrm{FA})$, and $\mathrm{A}^{\prime}$ were calculated from the summed data. As noted above, bias increased ( $B^{\prime \prime}$ decreased) across all conditions as a function of retention-interval duration. However, bias did not vary significantly as a function of the number of trials since the last nonmatch trial or the duration of Lag 1, and there was no interaction between these variables and retention-interval duration.

Quite a different picture was seen for the sensitivity index, $A^{\prime}$. Figure 5 contrasts sensitivity on $N M+1$ trials with that on the remaining trials of the session. The data in Figure 5 were obtained by combining the raw data into five categories as a function of the number of trials from the last nonmatch trial. These values were then used to calculate corresponding values of $P\left(\right.$ Hit), $P(F A)$, and $A^{\prime}$. Examination of the data revealed no significant differences between performance at distances of 3 to 5,6 to 10 , and 11 to 15 trials from the last nonmatch trial. Therefore, the data from these trials were further combined into a single category (NM +3 to 15$)$ for statistical analysis. Analysis of variance revealed significant effects of distance $[\mathbf{N M}+1$ vs. $\mathbf{N M}+2$ vs. $\mathbf{N M}+3$ to 15 ; $F(2,8)=106.93]$ and retention interval $[2.5$ vs. 5 vs. $10 \mathrm{sec}, \mathrm{F}(2,8)=8.83$ ] but no interaction between distance and retention interval. Thus, the accuracy of responding increased as a function of the number 


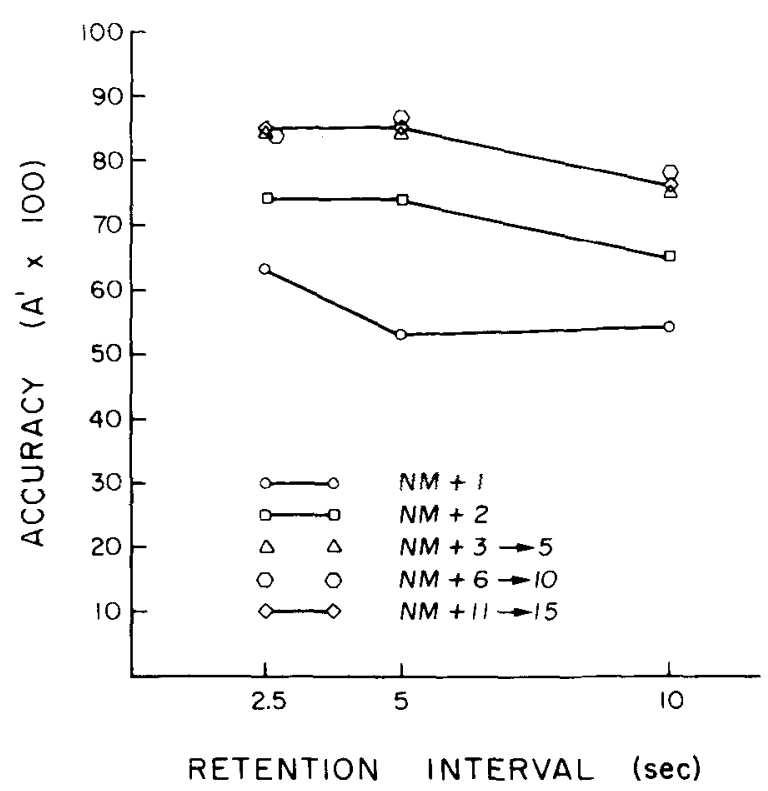

Figure 5. Accuracy $\left(A^{\prime}\right)$ as a function of the retention interval at distances of $1,2,3$ to 5,6 to 10 , and 11 to 15 trials from the last nonmatch trial. The data represent group means for the last 16 days of Experiment 2.

of trials from the last nonmatch trial, but the time course of retention remained unchanged. Accuracy decreased significantly as the retention interval increased from 2.5 to $10 \mathrm{sec}$, even for Trials NM +11 to $\mathrm{NM}+15[\mathrm{~F}(2,8)=5.95]$. Thus, even at the greatest distances from the last potentially interfering stimulus, response accuracy still decreased as a function of retention-interval duration.

Detailed examination of performance on $\mathrm{NM}+1$ trials indicated that the accuracy of responding varied significantly as a function of the duration of the retention interval $[F(1,4)=7.99]$ and Lag $1[F(1,4)=$ 7.85; see Figure 6]. The interaction between Lag 1 and the retention interval approached, but did not achieve, significance $[F(1,4)=6.26, p<.10]$. Thus, the time between the to-be-remembered stimulus and the previous, conflicting stimulus affected the overall accuracy of responding but had only a marginal effect on the time course of retention.

\section{Discussion}

The results of Experiment 2 indicate that interference from previous conflicting trials is an important determinant of the accuracy of responding in the CNM task. As predicted, accuracy increased as a function of the number of trials since the last nonmatch trial. Furthermore, accuracy on trials immediately following a nonmatch trial increased as a function of the duration of the interval (Lag 1) between the nonmatch trial and the previous conflicting trial. However, interference had little effect on the time course of retention in Experiment 2. Analysis of performance on NM +1 trials showed no interaction between Lag 1 and retention interval duration (although there was a trend in that direction). Similarly, there was no significant interaction between retention-interval duration and the number of trials since the last nonmatch trial. Finally, examination of performance on trials $\mathrm{NM}+11$ to 15 (the greatest possible number of trials from a potential conflicting stimulus) revealed that accuracy declined significantly as the retention interval increased from 2.5 to $10 \mathrm{sec}$. Thus, even under conditions of minimal PI, significant forgetting still occurred.

One potential problem in interpreting the data from Experiment 2 involves the trend toward increased bias (decreased B') $^{\prime \prime}$ at long retention intervals. This trend may indicate that sensitivity and bias are not independent in the CNM task; as sensitivity drops, bias increases. If so, this would represent a serious violation of the assumption of the theory of signal detection. More likely, however, this trend reflects an independent effect of long intertrial intervals on bias; as the interval increases, the subject becomes "impatient" and more apt to respond. Thus, bias increases. These alternative interpretations were examined in Experiment 3.

A second, potentially more serious problem is raised by a class of alternative hypothesis that at-

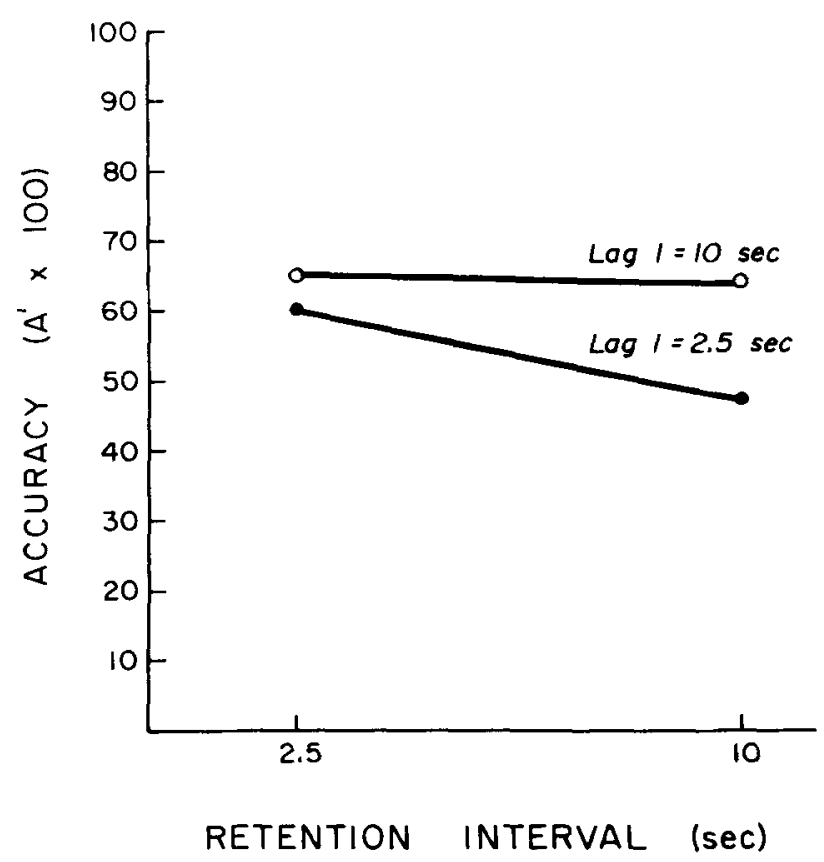

Figure 6. Accuracy on $N M+1$ trials as a function of the duration of the retention interval ( $\mathrm{Lag} 0$ ) and the time ( $\mathrm{Lag} 1)$ between the to-be-remembered nonmatch trial and the previous interfering stimulus. The points represent group means for the last 16 days of Experiment 2. 
tributes the poor performance on trials immediately following a nonmatch trial to the confounding effects of the early trial termination and delivery of reinforcement on the nonmatch trial. Thus, for example, it may be argued that the early termination of the nonmatch trial precludes adequate processing (encoding) of the trial stimulus, which, in turn, leads to poor performance when the nonmatch trial stimulus must be recalled. In this particular form, the alternative hypothesis is rather untenable, since nonmatch trials could not terminate until the stimulus had at least received sufficient processing to generate a correct response.

A more plausible form of this hypothesis is that the early trial termination and delivery of reinforcement disrupted rehearsal of the nonmatch trial stimulus. Such a disruption in rehearsal processing might contribute to the poor performance on $\mathrm{NM}+1$ trials. However, a disruption in rehearsal could not account for the effects of Lag 1 duration on Trial NM +1 accuracy (Figure 5) or for the poor performance on $\mathrm{NM}+2$ trials (Figure 4). Furthermore, it can be noted that pilot studies (Pontecorvo \& Heise, Note 1) indicate that the presentation of reinforcement for correct nonresponding on match trials does not reduce the overall accuracy of responding in the CNM task. These data further suggest that the occurrence of a stimulus change, and not the presentation of reinforcement, per se, is responsible for the poor performance on trials immediately following a nonmatch trial.

A final form of alternative hypothesis is that the early trial termination or delivery of reinforcement following a correct response on a nonmatch trial reduced attention to, and/or motivation on, the subsequent $\mathbf{N M}+1$ trial. This might account for the decreased accuracy both on Trial $\mathrm{NM}+1$ and on Trial NM +2 (where the unattended Trial NM +1 must be recalled). Experiment 3 was designed as a further test of this hypothesis.

\section{EXPERIMENT 3}

Experiment 3 was designed to control for effects on sensitivity and bias that were not specifically related to memory processes. The rats were trained to perform a simple (nonmemory) intensity discrimination task that was parametrically similar to the CNM task used in Experiment 2. There were 100 potentially reinforced "go" trials and 356 nonreinforced "nogo" trials per session. The intertrial interval (ITI, not a retention interval) varied within each session among the values $2.5,5$, and $10 \mathrm{sec}$. The relative intensities of the discriminative stimuli were manipulated to produce levels of response accuracy similar to those in Experiment 2. Because the task parameters and overall levels of performance were, therefore, equated in Experiment 2 (CNM, memory task) and in Experiment 3 (discrimination, nonmemory task), differences between the results of the two experiments could be attributed to differences in the memory requirements of the two tasks. Conversely, if a variable had similar effects in the two tasks, the effects could be attributed to processes common to both tasks (e.g., discrimination, attention, or motivation).

Of primary interest in Experiment 3 were the effects of ITI duration and distance (number of trials) from the last reinforced trial on response accuracy (A'). In Experiment 2, accuracy increased as a function of the number of trials since the last reinforced (nonmatch) trial, and performance on the trial following the reinforced trial depended on the time (Lag 1) between the reinforced trial and the previous (conflicting) trial. If, as was suggested, these effects were due to PI, a memory-dependent process, then they should have been evident only in the memory (CNM) task. If, on the other hand, these effects were due to some artifact (e.g., reduced attention or motivation following a reinforced trial), then similar effects should have occurred in the discrimination task (Experiment 3).

Also of interest in Experiment 3 was the relationship between ITI duration and bias ( $\left.\mathbf{B}^{\prime \prime}\right)$. If, as was hypothesized in Experiment 2, long ITIs caused the subjects to become impatient and thus led to an increase in bias, then long ITIs should have affected performance both in the CNM task and in this discrimination task. Alternatively, if the increase in bias at long retention intervals in Experiment 2 resulted from some task-specific interaction of sensitivity and bias, then the effect should not have been present in the discrimination task.

\footnotetext{
Method

Subjects. The subjects were five naive 90 -day-old rats obtained from the same supplier as the rats in Experiment 2.

Apparatus. During the first 15 days of Experiment 3, the apparatus was identical to that in Experiments 1 and 2. On Day 16 and thereafter, the intensity ratio of the dim stimulus to the bright stimulus was increased from 0.04 to 0.60 .

Procedure. All rats were first shaped to leverpress and then were trained to perform a simple (nonmemory) go/no-go intensity discrimination. For each animal, one stimulus was designated the positive stimulus and the other stimulus was designated the negative stimulus (balanced across animals). The first leverpress on a trial in which the positive stimulus was presented (a "go" trial) terminated the trial and produced water reinforcement. Leverpresses in the presence of the negative stimulus did not terminate the trial, were not reinforced, and caused the "no-go" trial to be repeated (correction trials). Failure to respond on either a go trial or a no-go trial had no scheduled consequence. The sequence of trials was arranged such that reinforcements were possible at the same point in the session as in the corresponding CNM procedure (i.e., go trials in the discrimination task and nonmatch trials in the CNM task were scheduled to occur at the same points in the session). Thus, there were 100 go and 358 no-go trials per session. The maximum trial duration was $5 \mathrm{sec}$.
} 
During the first 15 sessions, the discriminative stimuli were identical to those used in the CNM task (dim/bright $=0.04)$, and the ITI was fixed at $1 \mathrm{sec}$. After completing 15 sessions with these stimuli, the rats were retrained to perform the discrimination with a new, more difficult set of stimuli $(\mathrm{dim} /$ bright $=0.60)$. The duration of the ITI was gradually increased as the rats met performance criteria similar to those in Experiment 2. During the test phase of the experiment, the ITI varied within each session among the values 2.5, 5, and $10 \mathrm{sec}$. As in Experiment 2, all data analyses were based on Days 21-36 of this multiple ITI condition.

\section{Results}

All rats rapidly acquired the discrimination task. The mean value of $A^{\prime}$ for Days 11-15 was 0.94. Retraining with the second set of stimuli progressed more slowly. The rats gradually acquired the task, reaching the multiple ITI stage in a mean of 48 days.

Figure 7 shows the mean (group) values of $P(\mathrm{Hit})$ and $P(F A)$ as a function of the ITI. Both $P(\mathrm{Hit})$ and $P(F A)$ increased with ITI duration. Analysis of the corresponding values of $\mathbf{A}^{\prime}$ and $\mathbf{B}^{\prime \prime}$ indicated that $\mathbf{B}^{\prime \prime}$ decreased significantly (mean $B^{\prime \prime}$ s $=0.24,-0.05$, and -0.12 at the $2.5-, 5-$, and 10 -sec ITIs, respectively $[F(2,8)=19.72]$ but that $A^{\prime}$ did not change (mean $\mathrm{A}^{\prime} \mathrm{s}=0.84,0.83$, and 0.81 ) as ITI duration increased. Thus, increasing ITI duration increased the relative tendency to respond (bias) but did not affect the accuracy of responding in this discrimination task.

Performance was also analyzed as a function of the distance (number of trials) from the last go trial.

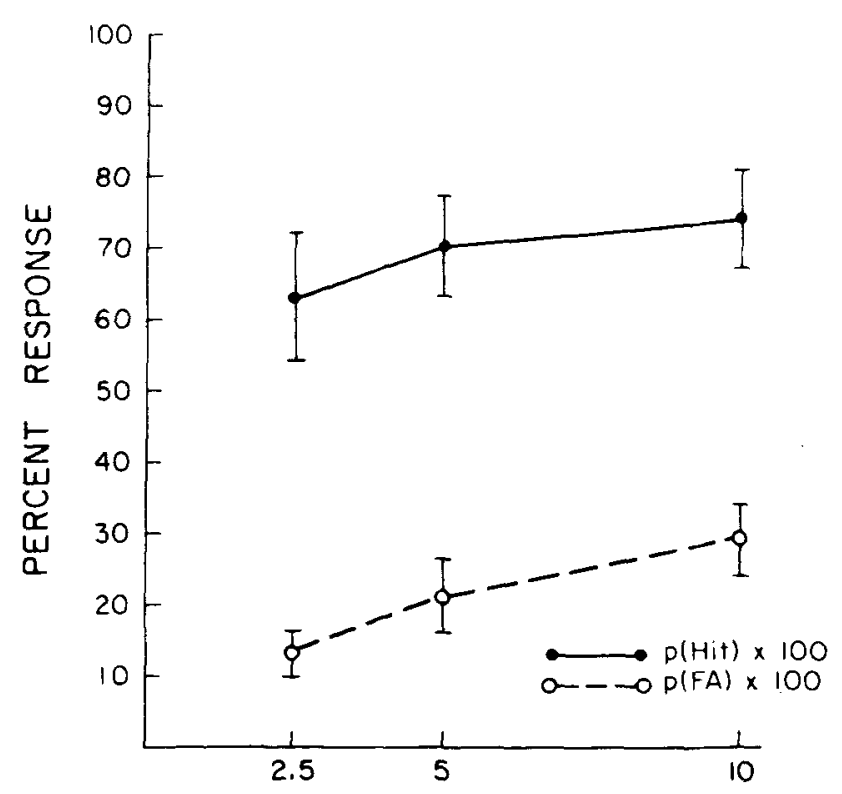

INTERTRIAL INTERVAL (sec)

Figure 7. Mean (group) values of $P($ Hit) and P(FA) as a function of the intertrial interval for the last 16 days of Experiment 3. Bars show standard error of the means.
As in Experiment 2, the raw data were summed over days as a function of distance, and values of $P(H i t)$, P(FA), and $A^{\prime}$ were then calculated. Preliminary examination of the data revealed no differences in $\mathbf{A}^{\prime}$ as a function of distance, so the data were regrouped into two categories for statistical comparison: trials following go trials and trials following no-go trials. Analysis of variance revealed no significant effects of trial type and no interaction of trial type with ITI duration. Thus, the number of trials since the last reinforced trial did not affect the accuracy of responding in this discrimination task.

\section{Discussion}

The results indicate that sensitivity in a simple discrimination task does not vary as a function of the number of trials since the last reinforced trial. This result is in direct contrast with the results obtained in Experiment 2 (CNM memory task), in which sensitivity was low on the trial immediately following a reinforced (nonmatch) trial and increased rapidly thereafter. This result confirms the assertion that the effects seen in Experiment 2 were related to the memory requirements of the task and is consistent with the hypothesis that interference from previous conflicting trials disrupted performance on the trials immediately following a nonmatch trial. Nonspecific factors, such as a reduction in attention or motivation resulting from the early termination of the trial or the delivery of reinforcement on a go (nonmatch) trial, cannot account for these results, since such factors would be expected to influence performance both in a memory (Experiment 2) and a discrimination (Experiment 3) task.

The results of Experiment 3 also indicate that increasing the duration of the ITI can affect the tendency to respond (bias) in a simple go/no-go discrimination task. This result suggests that the increase in bias seen at long ITIs (retention intervals) in the CNM task (Experiment 3 ) could result at least in part from nonmemory processes. Specifically, rats may be more apt to respond when a response opportunity is delayed for too long a time.

It is not clear whether these results can be generalized to other memory tasks. Analogous studies have produced similar results in go/no-go delayed-alternation tasks (Heise, Note 2). However, retention-interval duration should have little effect on responsivity in paired-trial tasks (e.g., Wallace et al., 1980), since the retention interval occupies a relatively small portion of the time between response opportunities. Although a number of studies have shown that extreme retention intervals can affect responsivity in pairedtrial tasks (D'Amato, 1973; Gleitman, Wilson, Herman, \& Rescorla, 1963), most studies report little effect of retention-interval duration on the overall tendency to respond (e.g., Honig \& Wasserman, 1981). On the other hand, Roberts and Kraemer (1982) 
have recently suggested that a pigeon's readiness to respond (as measured by pecking on the sample key) may increase as ITI duration increases. Together with the results of the present experiment, Roberts and Kraemer's results suggest that experimenters should be alert to possible changes in responsivity whenever the duration between response opportunities is manipulated.

\section{GENERAL DISCUSSION}

This paper introduced a new version of the delayed matching-to-sample paradigm-the continuous nonmatching-to-sample (CNM) task. Rats readily acquired the CNM task and performed reliably with retention intervals up to $10 \mathrm{sec}$ (the longest interval tested). The overall accuracy of responding and the degree of retention in these experiments were considerably better than those obtained by Wallace et al. (1980) in the only previous study of DMS performance by rats. There were, of course, numerous differences between the parameters (e.g., modality, duration of the stimuli, number of trials per session) of the Wallace et al. procedure and those used in the present study. Any of these parametric differences, along with more fundamental task differences (e.g., continuous vs. paired trials, nonmatching vs. matching) may have contributed to the superior performance in the present study. In any case, the present experiments clearly demonstrate the feasibility of the CNM task as a baseline for the study of short-term memory in rats. Because it is readily acquired and consistently performed, the CNM task offers promise for improving the cross-species generality of memory theories, for investigating the biological basis of memory (e.g., Pontecorvo \& Heise, Note 1), and for studying applied problems such as aging or exposure to toxic chemicals.

Analysis of the effects of interference from previous conflicting trials (PI) showed that PI affected the accuracy of responding in the CNM task. Accuracy was lowest on trials immediately following a nonmatch trial and increased rapidly thereafter. Thus, accuracy was lowest immediately following a stimulus change and increased as a function of the number of trials since the last conflicting stimulus (Figure 5). Furthermore, accuracy on the trial following a stimulus change increased as a function of the time (Lag 1) between the to-be-remembered nonmatch trial and the previous conflicting trial (Figure 6). Factors unrelated to memory could not account for these effects, since no effects of trials or intertrial interval ( $\mathrm{Lag} 1)$ were observed in an analogous discrimination task (Experiment 3).

Although PI was an important determinant of the accuracy of responding in the CNM task, PI did not affect the time course of retention. Neither the distance from the last nonmatch trial nor the duration of Lag 1 affected the rate of retention (Figures 5 and 6). Accuracy decreased as retention interval increased, even under conditions of minimal PI. It should be noted that this pattern of results is not an artifact of the particular subjects or experimental parameters used in the present experiments. Similar analyses have been performed on data from other experiments in our laboratory (e.g., Pontecorvo \& Heise, Note 1). In each case, overall accuracy increased, but the rate of forgetting (the rate of decline in accuracy with time) remained unchanged as a function of the number of trials since the last conflicting stimulus.

These results are clearly inconsistent with the temporal discrimination theories (e.g., D'Amato, 1973) described above. These theories hold that PI is a primary determinant of the time course of retention and thus would incorrectly predict that forgetting should be most rapid immediately following a stimulus change (i.e., on NM +1 trials) and be minimal at long distances from the last conflicting stimulus (Trials $\mathbf{N M}+11$ to $\mathrm{NM}+15$ ).

These results do not, of course, rule out the importance of temporal factors in short-term memory tasks. Indeed, the large effects of PI on response accuracy, and the fact that accuracy improved as a function of the time between the to-be-remembered and the previous conflicting stimuli, suggest that temporal confusion may be an important source of errors. Furthermore, it must be noted that PI has been shown to affect retention in other short-term memory tasks (e.g., Grant, 1975). Nevertheless, the failure of PI to alter retention in the present experiments indicates that temporal confusion does not necessarily increase with retention-interval duration. Confusion between stimuli cannot account for forgetting in this task; additional factors must be involved.

One obvious candidate is information loss due to intrinsic processes such as trace decay. Grant and Roberts (Grant, 1975; Roberts \& Grant, 1976) have proposed an elegant decay model that has been successful in predicting the effects of PI on pigeons' performance in most delayed matching-to-sample tasks. However, the Grant and Roberts model depends on the assumption that decay will be negatively accelerated; that is, it depends on the assumption that forgetting will be greatest in the first few seconds of each retention interval. Thus, the model cannot be applied to situations such as the present CNM task, in which more forgetting occurred between 5 and $10 \mathrm{sec}$ than between 0 and $5 \mathrm{sec}$.

Grant and Roberts' model could, of course, be modified to include a rehearsal mechanism, which might delay the onset of trace decay for some time. Indeed, a number of recent experiments (Brodigan \& Peterson, 1976; Grant, 1981b; Maki \& Hegvik, 1980 ) suggest the operation of this sort of mechanism in many short-term memory tasks. However, the in- 
clusion of a rehearsal mechanism deprives the Grant and Roberts model of its attractive elegance and predictive value. Moreover, since the observed rate of forgetting can no longer be directly linked to the underlying rate of trace decay, the addition of a rehearsal assumption minimizes the explanatory value of the decay concept. In light of these problems, it may be more profitable to abandon the decay model in favor of an alternative approach in which memory is seen as an active process of selection, storage, and retrieval, rather than a process of passive decay or confusion (see Grant, 1981a, for additional arguments to this effect).

In the context of this sort of model, PI would represent a more complex phenomenon than would be predicted from temporal discrimination on trace decay theories. Previous conflicting stimuli might interfere with performance in several different ways: by disrupting or altering stimulus selection or encoding, by competing for rehearsal time, and/or by providing conflicting information at the time of response output. Which process or processes are interfered with, and the ultimate consequence of this interference for the time course of retention likely depends on factors such as the stimulus parameters and task demands. For example, interference may be most likely to affect retention in situations in which rehearsal capacity is already taxed, that is, those in which the subjects are relatively inexperienced, the stimuli are relatively difficult, or memory for several different stimuli is required. On the other hand, effects on encoding processes are likely to depend on the nature of the stimuli and context of the stimulus presentation (e.g., Devine, Burke, \& Rohack, 1979; Grant, 1980; Reynolds \& Medin, 1981).

The challenge for future experiments, then, would be to investigate in more detail the relationships between these sorts of stimulus and task parameters and the effects of previous interfering events. Because it is rapidly acquired, readily analyzed, and sensitive to PI, the CNM task introduced in the present experiments appears well suited for addressing these sorts of issues.

\section{REFERENCE NOTES}

1. Pontecorvo, M. J., \& Heise, G. A. Effects of scopolamine on rat's continuous non-matching to sample performance. Paper presented at the meeting of the American Psychological Association, Washington, D.C., August 1982.

2. Heise, G. A. Personal communication, August 1981.

\section{REFERENCES}

Bradley, P. B., \& Roberts, M. H. T. A method for studying the effects of drugs on recent memory in animals. In M. Ya. Mikel'son \& V. G. Longo (Eds.), Pharmacology of conditioning, learning and retention. Oxford: Pergamon Press, 1965.
Brodigan, D. L., \& Peterson, G. B. Two-choice conditional discrimination performance of pigeons as a function of reward expectancy, prechoice delay, and domesticity. Animal Learning \& Behavior, 1976, 4, 121-124.

Chorazyna, H. Investigation of recent memory of acoustic stimuli in normal dogs. Bulletin of the Polish Academy of Sciences, 1959, 7, 119-121.

Christoph, G. R. Proactive interference and spatial alternation performance in rats: $A$ behavioral and psychopharmacological analysis. Unpublished doctoral dissertation, Indiana University, 1976.

D'Aм^то, M. R. Delayed matching and short-term memory in monkeys. In G. H. Bower (Ed.), The psychology of learning and motivation: Advances in research and theory (Vol. 7). New York: Academic Press, 1973.

D'Aмато, M. R., \& O'Neill, W. Matching behavior: Some methodological problems. Behavior Research Methods \& Instrumentation, 1970, 2, 162-164.

Devine, J. V., Burke, M. W., \& Rohack, J. J. Stimulus similarity and order as factors in visual short-term memory in nonhuman primates. Journal of Experimental Psychology: Animal Behavior Processes, 1979, 5, 335-354.

Fletcher, H. J. The delayed-response problem. In A. M. Schrier, H. F. Harlow, \& F. Stollnitz (Eds.), Behavior of nonhuman primates (Vol. 1). New York: Academic Press, 1965.

Fletcher, H. J., \& DAvis, J. K. Evidence supporting an intratrial interpretation of delayed response performance of monkeys. Perceptual and Motor Skills, 1965, 21, 735-742.

Gleitman, H., Wilson, W. A., Jr., Herman, M. M., \& Rescorla, R. A. Massing and within delay position as factors in delayed response performance. Journal of Comparative and Physiological Psychology, 1963, 56, 445-451.

Gordon, W. C., Brennan, M. J., \& Schlesinger, J. L. The interaction of memories in the rat: Effects on short-term retention performance. Learning and Motivation, 1976, 7, 406-417.

Grant, D. S. Proactive interference in pigeon short-term memory. Journal of Experimental Psychology: Animal Behavior Processes, 1975, 1, $207-220$.

Grant, D. S. Delayed alternation in the rat: Effect of contextual stimuli on proactive interference. Learning and Motivation, $1980,11,339-354$.

Grant, D. S. Short-term memory in the pigeon. In N. E. Spear \& R. R. Miller (Eds.), Information processing in animals: Memory mechanisms. Hillsdale, N.J: Erlbaum, 1981. (a)

Grant, D. S. Stimulus control of information processing in pigeon short-term memory. Learning and Motivation, 1981, 12, 19-39. (b)

Grant, D. S., \& Roberts, W. A. Trace interaction in pigeon short-term memory. Journal of Experimental Psychology, 1973, 101, 221-229.

Green, D. M., \& Swets, J. A. Signal detection theory and psychophysics. New York: Wiley, 1966.

Grie R, J. Nonparametric indexes for sensitivity and bias: Computing formulas. Psychological Bulletin, 1971, 75, 424-329.

Heise, G. A. Discrete trial analysis of drug action. Federation Proceedings, 1975, 34, 1898-1903.

Herman, L. M., \& Gordon, J. A. Auditory delayed matching in the bottlenose dolphin. Journal of the Experimental Analysis of Behavior, 1974, 21, 19-26.

Hodos, W. Non-parametric index of response bias for use in detection and recognition experiments. Psychological Bulletin, $1970,74,351-354$.

HonIG, W. K. Studies of working memory in the pigeon. In S. H. Hulse, H. Fowler, \& W. K. Honig (Eds.), Cognitive processes in animal behavior. Hillsdale, N.J: Erlbaum, 1978.

Honig, W. K., \& Wasserman, E. A. Performance of pigeons on delayed simple and conditional discriminations under equivalent training procedures. Learning and Motivation, 1981, 12, $140-170$. 
Jarvik, M. E., Goldfarb, T. L., \& Carley, J. L. Influence of interference on delayed matching in monkeys. Journal of Experimental Psychology, 1969, 81, 1-6.

Konorski, J. A. A new method of physiological investigation of recent memory in animals. Bulletin of the Polish Academy of Sciences, 1959, 7, 115-117.

MaKI, W. S., \& Heavik, D. K. Directed forgetting in pigeons. Animal Learning \& Behavior, 1980, 8, 567-574.

MAson, M., \& WiLson, M. Temporal differentiation and recognition memory for visual stimuli in rhesus monkeys. Journal of Experimental Psychology, 1974, 103, 383-390.

Medin, D. L., \& DAvis, R. T. Memory. In A. M. Schrier \& F. Stollnitz (Eds.), Behavior of non-human primates. New York: Academic Press, 1974.

Morse, S. H. Proactive effects of stimuli, delays, and response position during delayed matching from sample. Animal Learning \& Behavior, 1976, 4, 37-40.

Nelson, K. R., \& Wasserman, E. A. Temporal factors influencing the pigeon's successive matching-to-sample performance: Sample duration, intertrial interval and retention. Journal of the Experimental Analysis of Behavior, 1978, 30, 153-167.

OLton, D. S. Characteristics of spatial memory. In S. H. Hulse, H. Fowler, \& W. K. Honig (Eds.), Cognitive processes in animal behavior. Hillsdale, N.J: Erlbaum, 1978.

Pollack, I., \& Norman, D. A. A non-parametric analysis of recognition experiments. Psychonomic Science, 1964, 1, 125-126.

Reynolds, T. J., \& Medin, D. L. Stimulus interaction and between trials interference in monkeys. Journal of Experimental Psychology: Animal Behavior Processes, 1981, 7, 334-347.

Roberts, W. A. Distribution of trials and intertrial retention in delayed matching to sample with pigeons. Journal of Experi- mental Psychology: Animal Behavior Processes, 1980, 6, 217-237.

Roberts, W. A., \& Grant, D. S. Studies of short term memory in the pigeon using the delayed matching to sample procedure. In D. L. Medin, W. A. Roberts, \& R. T. Davis (Eds.), Processes of animal memory. Hillsdale, N.J: Erlbaum, 1976.

Robents, W. A., \& Kraemer, P. J. Some observations on the effects of intertrial interval and delay on delayed matching to sample in pigeons. Journal of Experimental Psychology: Animal Behavior Processes, 1982. 8. 342.393

SPEAR, N. E. The processing of memories: Forgetting and retention. Hillsdale, N.J: Erlbaum, 1978.

Stepien, L. S., \& Cordeau, J. P. Memory in monkeys for compound stimuli. American Journal of Psychology, 1960, 73, 388-395.

Wallace, J., Steinert, P. A., Scohie, S. R., \& Spear, N. E. Stimulus modality and short-term memory in rats. Animal Learning \& Behavior, 1980, 8, 10-16.

Wasserman, E. A. Successive matching-to-sample in the pigeon: Variations on a theme by Konorski. Behavior Research Methods \& Instrumentation, 1976, 8, 278-282.

Worsham, $\mathbf{R}$. W. Temporal discrimination factors in the delayed matching-to-sample task in monkeys. Animal Learning \& Behavior, 1975, 3, 93-97.

Zentall, T. R., \& Hogan, D. E. Memory in the pigeon: Proactive inhibition in a delayed matching task. Bulletin of the Psychonomic Society, 1974, 4, 109-112.

(Manuscript received February 23, 1983; accepted for publication May 11, 1983.) 